\title{
Comparison of transcatheter arterial chemoembolization with raltitrexed plus liposomal doxorubicin vs. tegafur plus pirarubicin for unresectable hepatocellular carcinoma
}

\author{
Rui Liao ${ }^{1 \#}$, Xing-Diao Zhang ${ }^{2 \#}$, Gui-Zhong Li $^{3 \#}$, Ke-Le Qin ${ }^{1}$, Xiong Yan $^{1}$ \\ ${ }^{1}$ Department of Hepatobiliary Surgery, the First Affiliated Hospital of Chongqing Medical University, Chongqing, China; ${ }^{2}$ Department of General \\ Surgery, Dazhou Central Hospital, Dazhou, China; ${ }^{3}$ Department of General Surgery, Beibei Traditional Chinese Medicine Hospital, Chongqing, \\ China \\ Contributions: (I) Conception and design: R Liao, XD Zhang, GZ Li, X Yan; (II) Administrative support: R Liao, X Yan; (III) Provision of study \\ materials or patients: R Liao, KL Qin, X Yan; (IV) Collection and assembly of data: R Liao, XD Zhang, GZ Li, X Yan; (V) Data analysis and \\ interpretation: R Liao, XD Zhang, GZ Li; (VI) Manuscript writing: All authors; (VII) Final approval of manuscript: All authors. \\ \#These authors contributed equally to this work. \\ Correspondence to: Xiong Yan. Department of Hepatobiliary Surgery, the First Affiliated Hospital of Chongqing Medical University, Chongqing, \\ China. Email: yxiong1964@aliyun.com.
}

Background: There is still no general consensus on the optimal chemotherapeutic agent selection for transcatheter arterial chemoembolization (TACE) in unresectable hepatocellular carcinoma (HCC). The present study aimed to compare the efficacy and safety of TACE with raltitrexed plus liposomal doxorubicin (R + PGLD) vs. tegafur plus pirarubicin $(\mathrm{T}+\mathrm{P})$ in patients with unresectable HCC.

Methods: A total of 148 patients with unresectable HCC treated with TACE between January 2012 and December 2016 were retrospectively analyzed. Of them, 74 patients were in the R + PGLD group and 74 patients were in the $\mathrm{T}+\mathrm{P}$ group (1:1). The treatment response of the tumor, overall survival (OS) time, and adverse effects were compared between the two groups.

Results: There were no significant differences in patient characteristics or embolization effect (lipiodol deposition) between the two groups $(\mathrm{P}>0.05)$. $\mathrm{R}+\mathrm{PGLD}$ treatment had a better clinical efficacy than $\mathrm{T}+\mathrm{P}$ treatment (OR: 64.9\% vs. 45.9\%, $\mathrm{P}=0.031$; DC: $89.2 \%$ vs. $74.3 \%, \mathrm{P}=0.032$ ). Portal vein invasion, hepatic vein invasion, tumor size and BCLC stage were associated with OR or DC after TACE using R + PGLD treatment. Survival analysis revealed that patients who received TACE with $\mathrm{R}+$ PGLD had a better prognosis than those treated with $\mathrm{T}+\mathrm{P}$. Moreover, some complications in the $\mathrm{R}+\mathrm{PGLD}$ group, including vomiting, myelosuppression and cardiotoxicity, were significantly lower than those in the $\mathrm{T}+\mathrm{P}$ group $(\mathrm{P}<0.05)$.

Conclusions: TACE with raltitrexed and liposomal doxorubicin could reduce the incidence of adverse reactions and significantly improve the OS of patients with unresectable HCC.

Keywords: Hepatocellular carcinoma (HCC); transcatheter arterial chemoembolization (TACE); raltitrexed; liposomal doxorubicin; prognosis

Submitted Feb 05, 2020. Accepted for publication Jul 08, 2020.

doi: 10.21037/jgo-20-59

View this article at: http://dx.doi.org/10.21037/jgo-20-59 


\section{Introduction}

Worldwide, hepatocellular carcinoma (HCC) is the sixth most prevalent cancer and ranks fourth in terms of cancerrelated mortality (1). Patients with early HCC may achieve a long-term complete response (CR) and improved survival after curative treatments $(1,2)$. Unfortunately, a majority of patients with HCC miss the opportunity for curative treatments at the time of diagnosis (3). Transcatheter arterial chemoembolization (TACE) has become the recommended palliative therapy for patients with intermediate- or advanced-stage HCC. However, the long-term outcomes of patients treated with TACE are still generally poor $(4,5)$.

Currently, there is still no general consensus on the optimal chemotherapeutic agent for TACE in unresectable HCC; thus, the effective management of TACE for these patients remains a major challenge $(6,7)$. To date, the commonly used drugs in TACE chemotherapy are fluorouracil (e.g., 5-fluorouracil and tegafur), platinum (e.g., carboplatin, cisplatin and oxaliplatin) and anthracycline (e.g., pirarubicin and doxorubicin). In theory, the combination of multiple drugs can improve efficacy and reduce tumor resistance through a variety of different medicinal mechanisms, but they simultaneously increase the side effects of drugs (8).

Raltitrexed is an antimetabolite folic acid analog and is part of a new generation of water-soluble thymidylate synthase (TS) inhibitors that directly inhibit TS, leading to DNA fragmentation and apoptosis and enhancing the interference of cancer cell DNA synthesis to increase antitumor activity (9). Recently, a retrospective study (10) suggested that TACE with oxaliplatin and raltitrexed could be effective with less toxicity in patients with advanced HCC with major portal vein tumor thrombus. Moreover, doxorubicin (DOX) monotherapy has long been used as one of the most important chemotherapeutic agents for HCC by directly destroying the DNA double helix structure and inhibiting DNA replication (11-13). By encapsulating doxorubicin hydrochloride contained within a liposome of polyethylene glycol (PEG), PEGylated liposomal doxorubicin (PGLD) can reduce DOX toxicity and block the drug recognized by the mononuclear macrophage system, thus prolonging its circulation, which is a new type of chemotherapy drug delivery system (12-14). To the best of our knowledge, the potential synergistic efficacy of TACE using raltitrexed plus PGLD in patients with unresectable HCC remains unclear and needs to be investigated.

In the present study, combination therapy with raltitrexed and PGLD in TACE could alleviate adverse chemotherapy responses in patients with HCC and improve overall survival (OS). Therefore, TACE with a combination of raltitrexed and PGLD may be a promising palliative therapeutic option for patients with unresectable HCC. We present the following article in accordance with the STROBE reporting checklist. Available at http://dx.doi. org/10.21037/jgo-20-59.

\section{Methods}

\section{Study cohorts}

This is a retrospective study conducted on a prospectively collected database on patients receiving TACE for unresectable HCC at the First Affiliated Hospital of Chongqing Medical University between January 2012 and December 2016. A total of 223 consecutive patients with unresectable HCC were enrolled in this study, who were treated with TACE using raltitrexed $(4 \mathrm{mg})$ plus PEG liposomal doxorubicin (40 mg, R + PGLD group) or tegafur (500-750 mg) plus pirarubicin (40 mg, $\mathrm{T}+\mathrm{P}$ group). The inclusion criteria were the following: (I) diagnosed with HCC by two types of typical imaging modalities and serological and pathological examinations; (II) valid and reliable laboratory test data; (III) no anticancer treatments; (IV) no history of other malignant diseases; (V) no serious organ dysfunction; (VI) aged 18 to 75 years; and (VII) no curative resection opportunity. There were 148 patients who qualified for the study and were divided into the $\mathrm{R}+$ PGLD group $(n=74)$ and $T+P$ group $(n=74)$. A total of 75 patients were excluded based on the above criteria. The deadline for follow-up was the end of December 2018. The study was conducted in accordance with the Declaration of Helsinki (as revised in 2013). The study was reviewed and approved by the Ethics Review Board of the First Affiliated Hospital of Chongqing Medical University (No. 2018107). The treatment options of TACE were fully discussed with the patients and a multidisciplinary discussion group (including radiologists, surgeons, and oncologists). Written consent was obtained from each patient before treatment.

\section{TACE procedures}

After local disinfection and anesthesia, the routinely used Seldinger method was used to perform the percutaneous puncture of the femoral artery in all patients. A selective 5 Fr catheter (Terumo Corporation, Tokyo, Japan) was 
introduced into the hepatic artery, and visceral angiography was performed to check the arterial blood supply to tumors. According to the patient's tumor location, size, and arterial supply, the tip of the catheter was introduced into the right or left hepatic artery or tumor-feeding artery. In some patients, a 2.9 Fr microcatheter (Terumo Corporation, Tokyo, Japan) was used for technical reasons. Hepatic artery infusion chemotherapy was injected into the tumor target vessel using raltitrexed plus liposomal doxorubicin or tegafur plus pirarubicin. Finally, the target vessel was fully embolized using $5 \mathrm{~mL}$ of hot lipiodol. All procedures were technically successful. After 4 weeks of treatment, the results of angiographic reexamination, which showed whether there was collateral circulation and iodized oil deposition, were used to determine whether repeated TACE should be performed.

\section{Postoperative evaluation}

To comparatively analyze the effectiveness of TACE in patients with unresectable HCC, the following clinical parameters were assessed: age, sex, hepatitis and cirrhosis history, alpha-fetoprotein, tumor number, tumor size, tumor stage, portal vein invasion, hepatic vein invasion, Eastern Cooperative Oncology Group (ECOG) performance status and laboratory indexes.

All patients were examined by upper abdominal enhanced CT after the TACE procedure at approximately 1 month after initial treatment. The modified Response Evaluation Criteria in Solid Tumors (mRECIST) (15) was used to assess the efficacy. In brief, CR was defined as no intratumoral arterial enhancement; partial response (PR) was defined as $<70 \%$ residual arterial enhancement in the target nodules; stable disease (SD) was defined as not qualifying for either PR or progressive disease (PD); $\mathrm{PD}$ was defined as an increased size of the treated tumor by $20 \%$, the interval development of new intrahepatic tumors, or both; and metastatic disease (MD) was defined as the detection of new extrahepatic tumors. The degree of remission was evaluated by the objective response $(\mathrm{OR}=$ $\mathrm{CR}+\mathrm{PR})$ and disease control $(\mathrm{DC}=\mathrm{CR}+\mathrm{PR}+\mathrm{SD})$. The ratio of the maximum cross-sectional diameter of iodized oil deposition to the largest tumor cross-section diameter was divided into three types: type I: dense $(\geq 70 \%)$; type II: partial filling (30-70\%); and type III: sparse ( $\leq 30 \%)$.

Adverse events and TACE-associated complications during and post treatment were recorded, including cardiotoxicities, vomiting, diarrhea, myelosuppression, and liver function damage.

\section{Statistical methods}

All statistical analyses were performed using SPSS 24.0 (SPSS, Inc., Chicago, IL, USA). Continuous variables are reported as the mean \pm standard deviation $(\mathrm{SD})$ in $\mathrm{OR}$ and DC analysis (Tables 1,2) or median (range) in characteristics analysis (Table 3), and were compared using Student's t test or the nonparametric Mann-Whitney $U$ test. Median of continuous variables were used as cut-off values. Categorical variables are expressed as a percentage and were examined using the $\chi^{2}$ test or Fisher's exact test. The survival curves were constructed by the Kaplan-Meier method and compared by the log rank test. A two-tailed $\mathrm{P}<0.05$ was considered significant.

\section{Results}

\section{Patient characteristics}

The baseline characteristics of the patients are described in Table 3. The $\mathrm{R}+\mathrm{PGLD}$ group and $\mathrm{T}+\mathrm{P}$ group comprised 61 and 64 men with a median age of 50.6 and 52.5 years, respectively. In the two groups, the majority of patients had cirrhosis (R + PGLD group and T + P group: $82.4 \%$ and $91.9 \%$, respectively) and liver function with Child-Pugh Grade A (R + PGLD group and T + P group: $89.2 \%$ and $97.3 \%$, respectively). Most patients had multiple tumors ( $\mathrm{R}+$ PGLD group and $\mathrm{T}+\mathrm{P}$ group: $77.0 \%$ and $67.6 \%$, respectively). Almost half of the patients had a large tumor size ( $\geq 5 \mathrm{~cm}$; $\mathrm{R}+\mathrm{PGLD}$ group and $\mathrm{T}+\mathrm{P}$ group: $47.3 \%$ and $48.6 \%$, respectively). Hepatic vein invasion ( $\mathrm{R}+\mathrm{PGLD}$ group and $\mathrm{T}+\mathrm{P}$ group: $28.4 \%$ and $21.6 \%$, respectively) and portal vein invasion (37.8\% in both groups) occurred in approximately one-fourth and one-third of patients in the $\mathrm{R}$ + PGLD group and T + P group, respectively. Additionally, in the $\mathrm{R}+\mathrm{PGLD}$ group and $\mathrm{T}+\mathrm{P}$ group, 6 (8.1\%) and 15 (20.3\%) patients were BCLC stage A, and $33(44.6 \%)$ and $24(32.4 \%)$ patients were BCLC stage B, respectively. Both groups had 35 (47.3\%) patients who were BCLC stage C. The baseline clinic pathological characteristics were broadly similar between the two groups $(\mathrm{P}>0.05)$.

\section{Treatment response of patients post TACE treatment}

The posttreatment response was evaluated by CT as shown 
Table 1 Subgroups analysis of OR and DC achievement in R + PGLD group

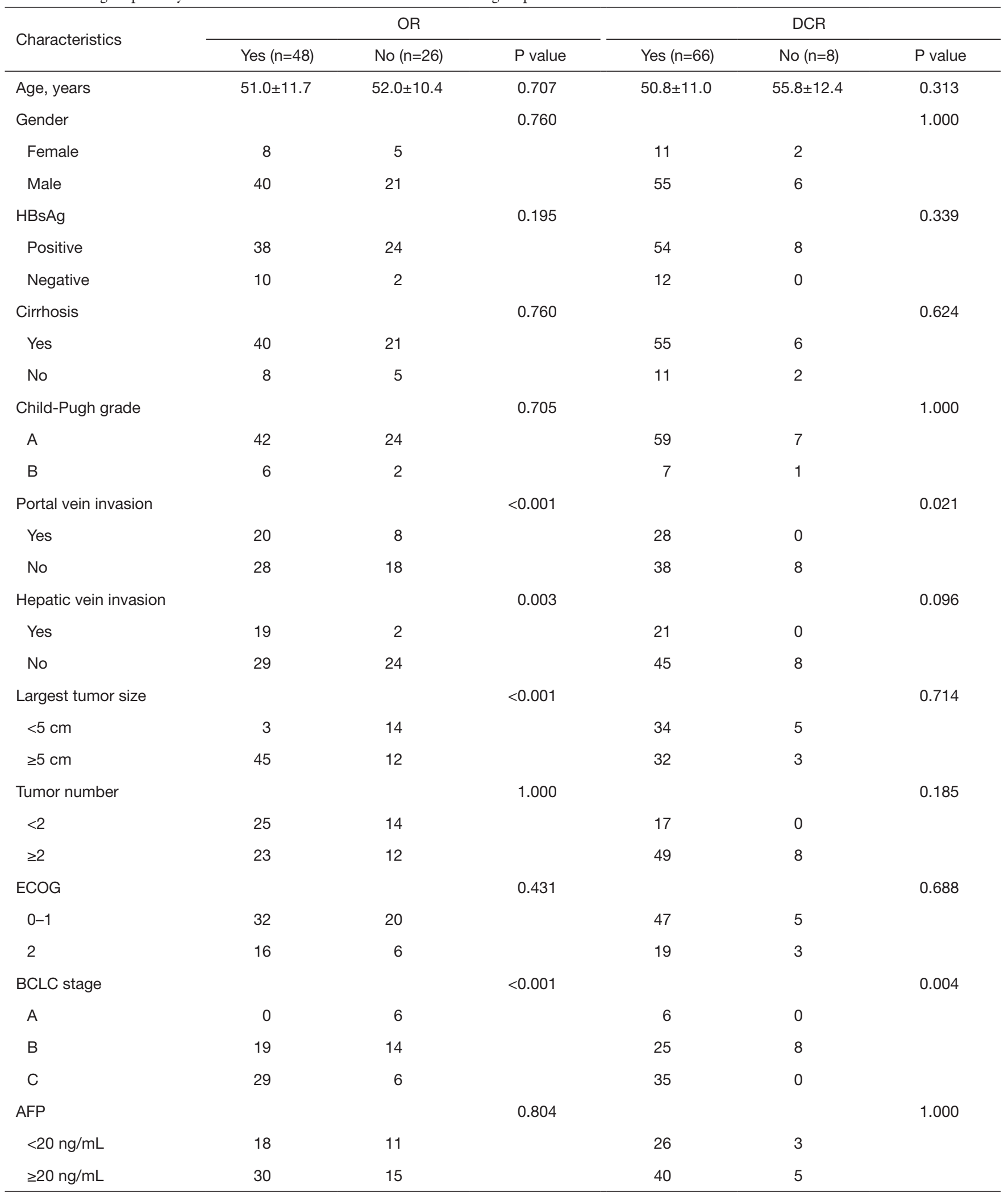

Table 1 (continued) 
Table 1 (continued)

\begin{tabular}{|c|c|c|c|c|c|c|}
\hline Characteristics & \multicolumn{3}{|c|}{ OR } & \multicolumn{3}{|c|}{ DCR } \\
\hline WBC, $10^{9} / \mathrm{L}$ & $5.7 \pm 2.3$ & $6.1 \pm 2.5$ & 0.475 & $5.8 \pm 2.4$ & $6.2 \pm 2.0$ & 0.620 \\
\hline PLT, $10^{\circ} / \mathrm{L}$ & $132.8 \pm 48.6$ & $155.1 \pm 56.7$ & 0.097 & $141.8 \pm 54.0$ & $131.4 \pm 37.1$ & 0.496 \\
\hline ALB, g/L & $44.4 \pm 4.8$ & $44 \pm 5.1$ & 0.744 & $44.4 \pm 4.9$ & $43.8 \pm 4.4$ & 0.722 \\
\hline ALT, U/L & $48.9 \pm 30.2$ & $47.0 \pm 26.9$ & 0.787 & $47.9 \pm 29.0$ & $51.1 \pm 30.3$ & 0.782 \\
\hline AST, U/L & $49.4 \pm 27.4$ & $47.0 \pm 26.9$ & 0.125 & $47.3 \pm 27.1$ & $37.1 \pm 12.7$ & 0.087 \\
\hline
\end{tabular}

OR, objective response; $D C$, disease control; $R+P G L D$, raltitrexed plus polyethylene glycol liposomal doxorubicin; HBsAg, hepatitis $B$ virus surface antigen; ECOG, Eastern Cooperative Oncology Group; BCLC, Barcelona Clinic Liver Cancer; AFP, alpha fetoprotein; WBC, white blood cell; PLT, platelet; ALB, albumin; TBIL, total bilirubin; ALT, alanine aminotransferase; AST, aspartate aminotransferase.

Table 2 Subgroups analysis of OR and DC achievement in $\mathrm{T}+\mathrm{P}$ group

\begin{tabular}{|c|c|c|c|c|c|c|}
\hline Characteristics & \multicolumn{3}{|c|}{ OR } & \multicolumn{3}{|c|}{ DC } \\
\hline Age, years & $51.3 \pm 13.2$ & $53.55 \pm 11.8$ & 0.451 & $53.2 \pm 12.2$ & $50.6 \pm 13.3$ & 0.467 \\
\hline Gender & & & 0.171 & & & 1.000 \\
\hline Female & 7 & 3 & & 8 & 2 & \\
\hline HBsAg & & & 0.147 & & & 0.746 \\
\hline Positive & 25 & 35 & & 45 & 15 & \\
\hline Negative & 9 & 5 & & 10 & 4 & \\
\hline Cirrhosis & & & 1.000 & & & 0.643 \\
\hline Child-Pugh grade & & & 1.000 & & & 0.450 \\
\hline$A$ & 33 & 39 & & 54 & 18 & \\
\hline B & 1 & 1 & & 1 & 1 & \\
\hline Portal vein invasion & & & 0.057 & & & 0.785 \\
\hline Yes & 17 & 11 & & 20 & 8 & \\
\hline No & 17 & 29 & & 35 & 11 & \\
\hline Hepatic vein invasion & & & 0.002 & & & 1.000 \\
\hline Yes & 13 & 3 & & 12 & 4 & \\
\hline
\end{tabular}

Table 2 (continued) 
Table 2 (continued)

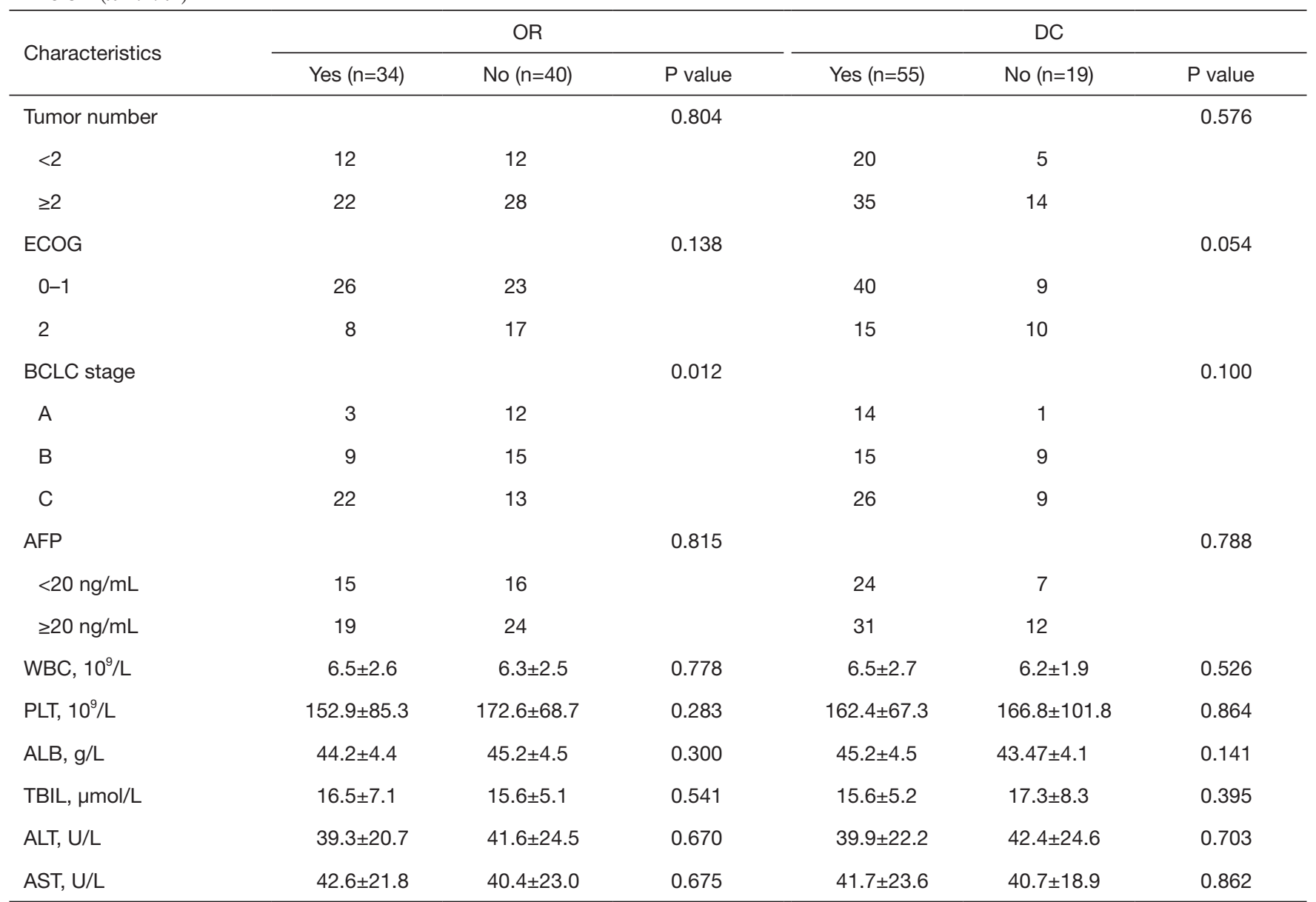

OR, objective response; DC, disease control; $T+P$, tegafur plus pirarubicin; HBsAg, hepatitis B virus surface antigen; ECOG, Eastern Cooperative Oncology Group; BCLC, Barcelona Clinic Liver Cancer; AFP, alpha fetoprotein; WBC, white blood cell; PLT, platelet; ALB, albumin; TBIL, total bilirubin; ALT, alanine aminotransferase; AST, aspartate aminotransferase.

in Table 4. There was no significant difference in lipiodol deposition (embolization effect) between the two groups $(\mathrm{P}=0.785)$. The rates of $\mathrm{OR}(64.9 \%$ vs. $45.9 \%)$ and $\mathrm{DC}$ (89.2\% vs. $74.3 \%)$ were better in the R + PGLD group than in the $\mathrm{T}+\mathrm{P}$ group $(\mathrm{P}=0.031$ and 0.032 , respectively). In the $\mathrm{R}+\mathrm{PGLD}$ group and $\mathrm{T}+\mathrm{P}$ group, 8 (10.8\%) and $4(5.4 \%)$ patients achieved CR, and 40 (54.1\%) and 30 (40.5\%) achieved PR, respectively. According to CT detection, in the $\mathrm{R}+\mathrm{PGLD}$ group and $\mathrm{T}+\mathrm{P}$ group, 2 and 7 patients had extrahepatic metastasis, respectively.

\section{Subgroup analysis of $O R$ and $D C$ in patients following TACE}

According to the different chemotherapy agents used for TACE, we compared the OR and DC in patients with unresectable HCC. In patients who underwent TACE with $\mathrm{R}+\mathrm{PGLD}$ treatment (Table 1), both portal vein invasion $(\mathrm{P}<0.001$ and 0.021 , respectively) and BCLC stage $(\mathrm{P}<0.001$ and 0.004 , respectively) were associated with $\mathrm{OR}$ and DC. Moreover, hepatic vein invasion $(\mathrm{P}=0.003)$ and tumor size $(\mathrm{P}<0.001)$ were also associated with $\mathrm{OR}$. After TACE with $\mathrm{R}+\mathrm{PGLD}$ treatment, patients with large tumors, no portal/hepatic vein invasion, and intermediate/ advanced BCLC stages tended to respond more than patients with small tumors, portal/hepatic vein invasion, and early BCLC stages. However, in patients after TACE with $\mathrm{T}+\mathrm{P}$ treatment (Table 2), only hepatic vein invasion $(\mathrm{P}=0.002)$ and BCLC stage $(\mathrm{P}=0.012)$ were related to $\mathrm{OR}$. No statistically significant difference was found between the clinical characteristics and DC (all $\mathrm{P}>0.05$ ). 
Table 3 Baseline characteristics of patients with unresectable HCC

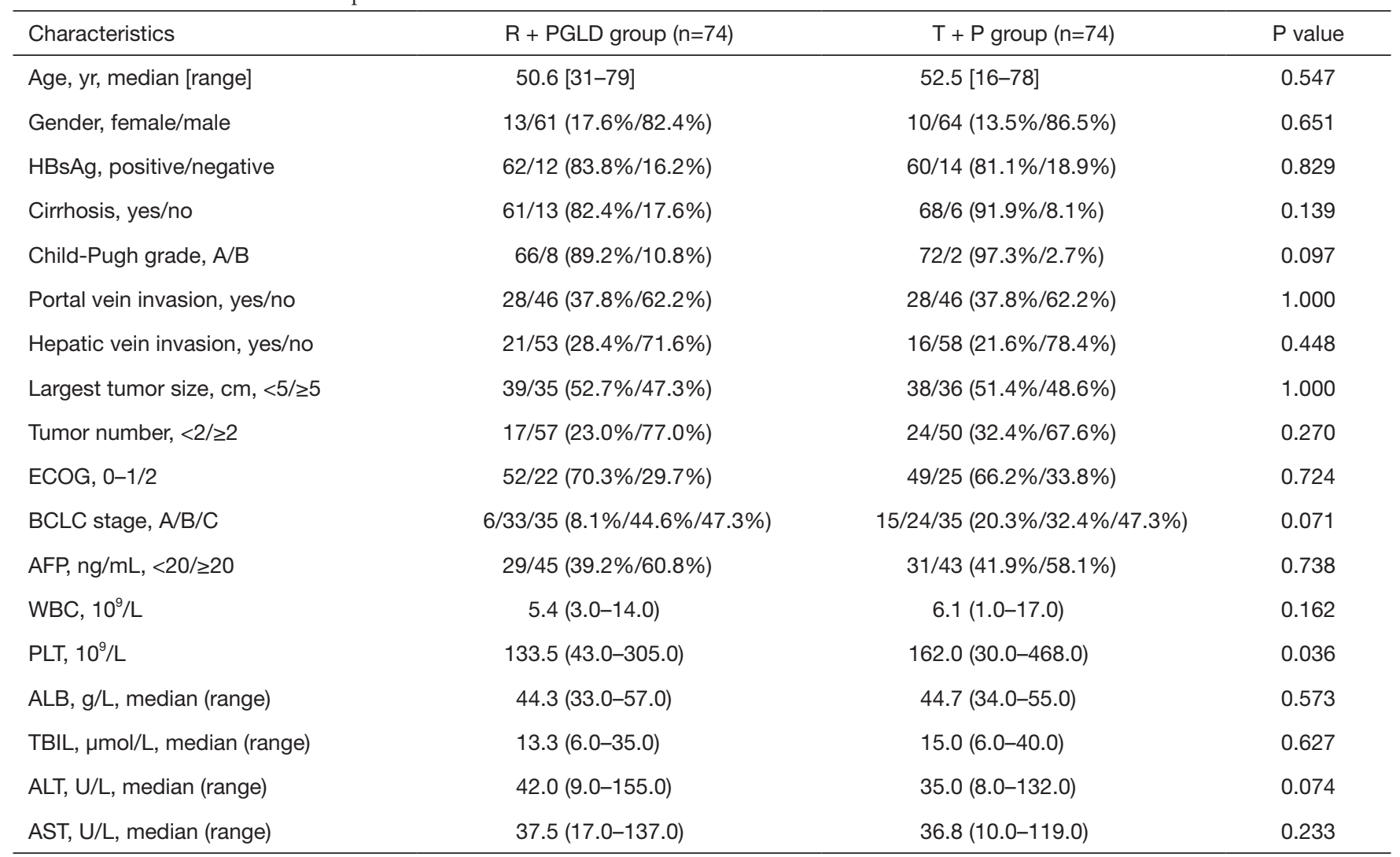

HCC, hepatocellular carcinoma; R + PGLD, raltitrexed plus polyethylene glycol liposomal doxorubicin; $T+P$, tegafur plus pirarubicin; HBsAg, hepatitis B virus surface antigen; ECOG, Eastern Cooperative Oncology Group; BCLC, Barcelona Clinic Liver Cancer; AFP, alpha fetoprotein; WBC, white blood cell; PLT, platelet; ALB, albumin; TBIL, total bilirubin; ALT, alanine aminotransferase; AST, aspartate aminotransferase.

Table 4 Treatment response of patients evaluated by CT

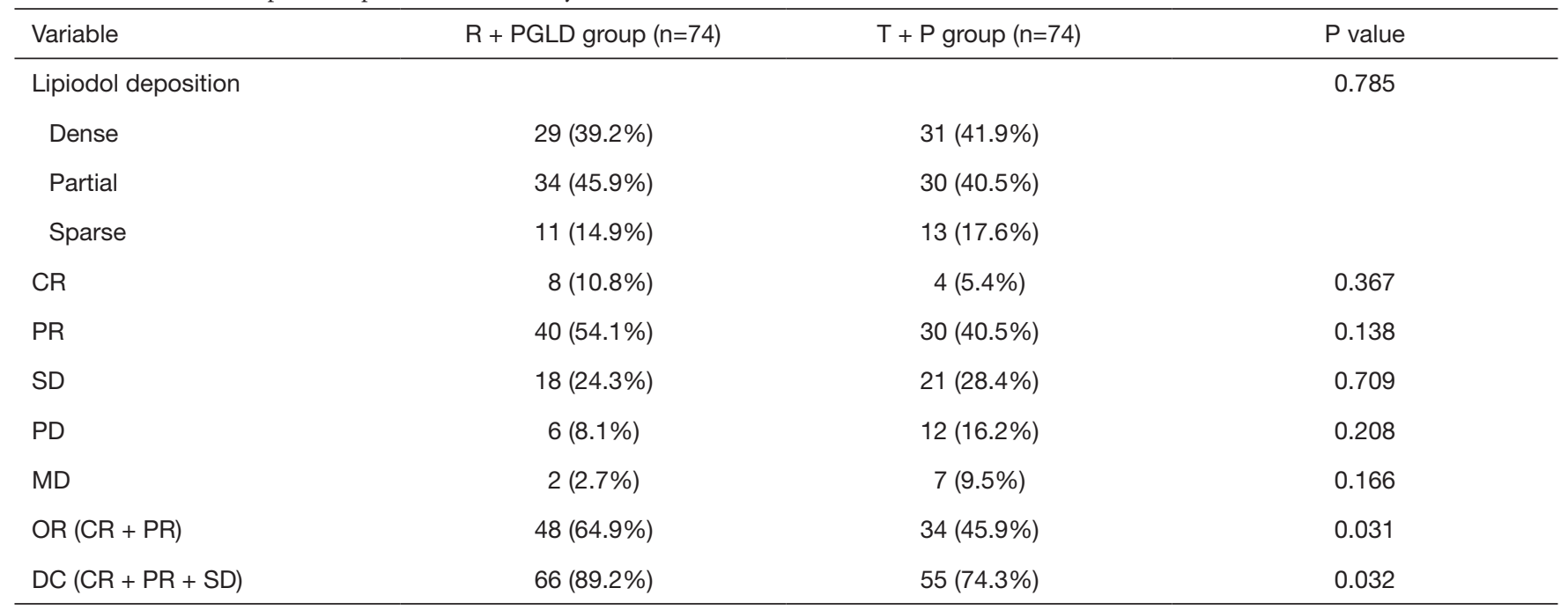

$\mathrm{R}+\mathrm{PGLD}$, raltitrexed plus polyethylene glycol liposomal doxorubicin; $\mathrm{T}+\mathrm{P}$, tegafur plus pirarubicin; CR, complete response; SD, stable disease; PD, progressive disease; MD, Metastatic disease; OR, objective response; DC, disease control. 
Table 5 Factors predicting overall survival by logistic regression analysis

\begin{tabular}{|c|c|c|c|c|}
\hline Variables & \multicolumn{2}{|c|}{ Univariate analysis } & \multicolumn{2}{|c|}{ Multivariate analysis } \\
\hline Age $(<51 / \geq 51$ years $)$ & $1.253(0.838-1.872)$ & 0.266 & - & NA \\
\hline Gender (female/male) & $0.884(0.516-1.515)$ & 0.651 & - & NA \\
\hline Cirrhosis (yes/no) & $0.689(0.368-1.290)$ & 0.236 & - & NA \\
\hline Child-Pugh grade (A/B) & $0.991(0.456-2.151)$ & 0.981 & - & NA \\
\hline Portal vein invasion (yes/no) & $3.107(2.004-4.816)$ & $<0.001$ & - & 0.623 \\
\hline Hepatic vein invasion (yes/no) & $2.738(1.677-4.471)$ & $<0.001$ & - & 0.182 \\
\hline Largest tumor size $(<5 / \geq 5 \mathrm{~cm})$ & $1.374(0.915-2.061)$ & 0.121 & - & NA \\
\hline BCLC stage $(A / B / C)$ & $2.660(1.886-3.751)$ & $<0.001$ & $2.509(1.788-3.519)$ & $<0.001$ \\
\hline $\operatorname{AFP}(<20 / \geq 20 \mathrm{ng} / \mathrm{mL})$ & $1.015(0.671-1.534)$ & 0.944 & - & NA \\
\hline WBC $\left(<5.8 / \geq 5.8 \times 10^{9} / L\right)$ & $0.778(0.522-1.160)$ & 0.213 & - & NA \\
\hline $\operatorname{PLT}\left(<143.0 / \geq 143.0 \times 10^{9} / \mathrm{L}\right)$ & $1.035(0.691-1.549)$ & 0.867 & - & NA \\
\hline ALB $(<40.0 / \geq 40.0 \mathrm{~g} / \mathrm{L})$ & $0.708(0.473-1.059)$ & 0.088 & - & NA \\
\hline TBIL (<14.7/ $\geq 14.7 \mu \mathrm{mol} / \mathrm{L})$ & $0.873(0.583-1.307)$ & 0.504 & - & NA \\
\hline $\operatorname{ALT}(<39.0 / \geq 39.0 \mathrm{U} / \mathrm{L})$ & $0.749(0.501-1.121)$ & 0.155 & - & NA \\
\hline AST $(<37.0 / \geq 37.0 \mathrm{U} / \mathrm{L})$ & $0.873(0.585-1.303)$ & 0.503 & - & NA \\
\hline
\end{tabular}

HBsAg, hepatitis B virus surface antigen; ECOG, Eastern Cooperative Oncology Group; BCLC, Barcelona Clinic Liver Cancer; AFP, alpha fetoprotein; WBC, white blood cell; PLT, platelet; ALB, albumin; TBIL, total bilirubin; ALT, alanine aminotransferase; AST, aspartate aminotransferase; TACE, transcatheter arterial chemoembolization; R + PGLD, raltitrexed plus polyethylene glycol liposomal doxorubicin; $\mathrm{T}+\mathrm{P}$, tegafur plus pirarubicin; OR, objective response; $\mathrm{DC}$, disease control.

\section{OS analysis}

In the $\mathrm{R}+\mathrm{PGLD}$ group, the mean OS was 16.2 months (range, 3.5-36.0 months). The 1-, 2-, and 3-year OS rates were $78.3 \%, 22.3 \%$ and $16.1 \%$, respectively. In the $\mathrm{T}$ $+\mathrm{P}$ group, the mean OS was 12.8 months (range, 3.036.0 months). The 1-, 2-, and 3 -year OS rates were $61.1 \%$, $15.2 \%$ and $6.9 \%$, respectively. Moreover, univariate analyses showed that portal/hepatic vein invasion (both $\mathrm{P}<0.001)$, tumor number $(\mathrm{P}<0.001)$, BCLC stage $(\mathrm{P}<0.001)$, therapeutic agents of TACE $(\mathrm{P}=0.016)$, OR $(\mathrm{P}<0.001)$ and DC $(\mathrm{P}=0.016)$ were associated with OS. After univariate analyses of our data, multivariate analyses were performed on significant clinical factors and demonstrated that BCLC stage $(\mathrm{P}<0.001)$ and therapeutic agents of TACE $(\mathrm{P}=0.029)$ were independent prognostic factors of OS (Table 5). Furthermore, compared with the patients treated by TACE using tegafur plus pirarubicin, the patients receiving TACE with raltitrexed plus liposomal doxorubicin treatments had better outcomes ( $\mathrm{P}=0.016$, Figure 1$)$.

\section{Adverse response of patients after TACE}

As listed in Table 6, pain and fever were the most common complications post-treatment. Compared with patients 


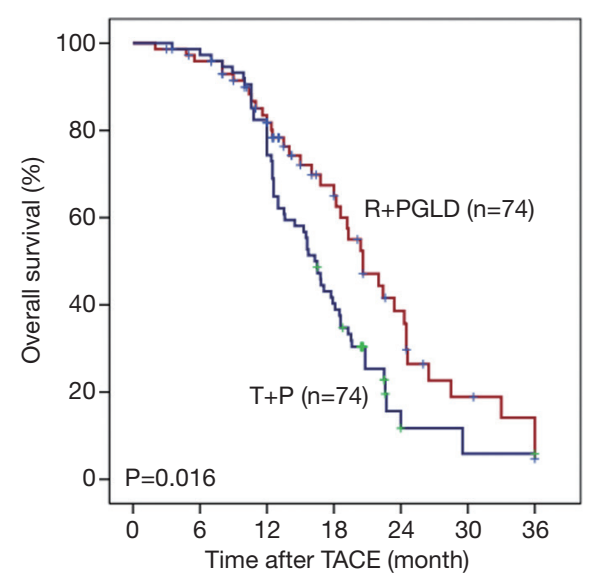

Figure 1 Overall survival comparison of TACE with raltitrexed plus liposomal doxorubicin $v s$. tegafur plus pirarubicin for unresectable HCC. TACE, transcatheter arterial chemoembolization; HCC, hepatocellular carcinoma.

in the $\mathrm{R}+\mathrm{PGLD}$ group, more complications happened in patients in the tegafur plus pirarubicin group, such as vomiting $(\mathrm{P}=0.011)$, myelosuppression $(\mathrm{P}=0.011)$ and cardiotoxicity $(\mathrm{P}=0.037)$. Here, myelosuppression was monitored according to routine blood tests (e.g., decreased counts of white blood cells, red blood cells and platelets). The evaluation indexes of cardiotoxicity included prolonged QT interval, increased cardiac troponin $\mathrm{T}(\mathrm{c} T \mathrm{Tn} \mathrm{T})$ and decreased left ventricular ejection fraction. In addition, liver function impairment was also observed in both groups. There were 11 and 8 patients with elevated transaminases (>5 ULN) and 9 and 10 patients with elevated bilirubin ( $>3 \mathrm{ULN}$ ) in the $\mathrm{R}+\mathrm{PGLD}$ group and $\mathrm{T}+\mathrm{P}$ group, respectively. There was no significant difference in liver function between the two groups $(\mathrm{P}>0.05)$, and none of the patients died from hepatic dysfunction.

\section{Discussion}

In our study, the patient characteristics and embolization effect (standard lipiodol deposition types) were broadly similar between the two groups. We discovered that: (I) $\mathrm{R}+\mathrm{PGLD}$ treatment had a better efficacy than $\mathrm{T}+\mathrm{P}$ treatment in HCC patients (OR: 64.9\% vs. $45.9 \%, \mathrm{P}=0.031$; DC: $89.2 \%$ vs. $74.3 \%, \mathrm{P}=0.032$ ). (II) Subgroup analysis showed that patients with portal vein invasion $(\mathrm{P}<0.001$ and $=0.021$ for $\mathrm{OR}$ and $\mathrm{DC}$, respectively) and hepatic vein invasion $(\mathrm{P}=0.003$ for $\mathrm{OR})$ were associated with worse $\mathrm{OR}$ or DC after TACE with $\mathrm{R}+\mathrm{PGLD}$ treatment. $\mathrm{R}+\mathrm{PGLD}$ treatment had a better OR or DC for HCC patients with large tumors $(\mathrm{P}<0.001$ for $\mathrm{OR})$ and higher BCLC stages $(\mathrm{P}<0.001$ for $\mathrm{OR}$ and $\mathrm{P}=0.004$ for $\mathrm{DC})$. In the $\mathrm{T}+\mathrm{P}$ treatment group, we found that only hepatic vein invasion and BCLC stage were related to OR. (III) OS analysis suggested that patients receiving TACE with raltitrexed plus liposomal doxorubicin treatment had better outcomes than those treated by TACE with tegafur plus pirarubicin. (IV) Compared with the $\mathrm{T}+\mathrm{P}$ group, some light and moderate common adverse events in the R + PGLD group were observed, such as vomiting, myelosuppression and cardiotoxicity.

So far, there are no consensus recommendation standards regarding the combination of chemotherapeutic agents for TACE. Here, it seems that combined raltitrexed and liposomal doxorubicin had a better response in inoperative HCC patients than treatment by tegafur and pirarubicin. In particular, the synergism between raltitrexed and liposomal doxorubicin is partially due to the dual destructive effects on the DNA of cancer cells, which could inhibit DNA synthase (raltitrexed) (9) and replication (doxorubicin) (11). Moreover, compared to conventional TACE, PEGylated liposomes, which have a higher efficacy for drug delivery, could improve the pharmacokinetics and reduce the chance of internalization by tumor cells (16).

Traditionally, TACE has not been used in cases of portal vein invasion due to its high embolic effect and the potential for worsening liver dysfunction. Additionally, our results showed that patients with portal vein invasion were associated with worse OR or DC after TACE regardless of $\mathrm{R}+\mathrm{PGLD}$ or $\mathrm{T}+\mathrm{P}$. However, TACE with R + PGLD had a better response at a higher BCLC stage and could be safely performed in these patients without significant hepatic function destruction. Notably, some studies indicated that TACE may prolong survival for patients with advanced HCC with portal vein invasion via selected therapeutic agents $(10,17,18)$. Here, all patients with vascular invasion are limited to a venous branch, and radioembolization and systemic therapy such as sorafenib are recommended for these patients. Further investigation is needed to help clinicians provide a viable option for patients with vascular invasion. Our results revealed that patients in the $\mathrm{R}+\mathrm{PGLD}$ group tended to present a better response for large tumors. One potential mechanism to explain this result is that TACE with $\mathrm{R}+$ PGLD might more easily contribute to the obvious necrosis (most lipiodol deposition types were dense and partially filled) of large tumors than 
Table 6 Adverse effects of two groups

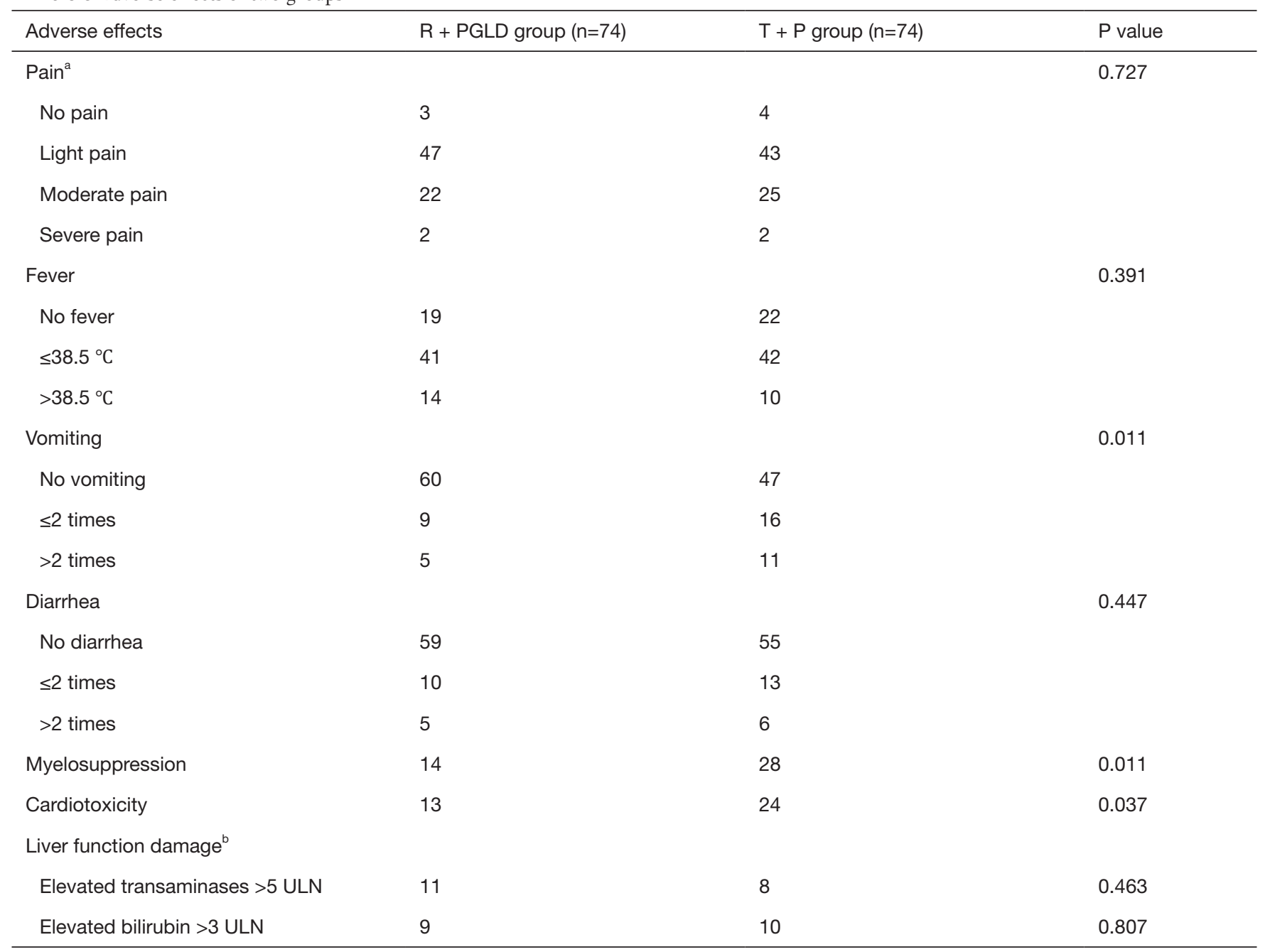

aPain VAS, pain visual analogue scale. No pain: pain VAS score =0; light pain: pain VAS score =1-3; moderate pain: pain VAS score =4-6; severe pain: pain VAS score $=7-10 .{ }^{b}$, Common Terminology Criteria for Adverse Events (CTCAE). $R+$ PGLD, raltitrexed plus polyethylene glycol liposomal doxorubicin; T + P, tegafur plus pirarubicin; ULN, upper limit of normal.

that of small tumors through superselective embolization of tumor-feeding arteries and sustained PEGylated liposome delivery in the large tumor tissue. Nevertheless, we could not exclude the possibility of patient selection bias because most patients were BCLC B and C stages. In this study, OR was also exhibited in the patients with BCLC-C. On one side, TACE could benefit for survival improvement of HCC patients via controlling the growth of tumor per se (19). On the other side, all patients with vascular invasion received radio-embolization and systemic therapy (e.g., sorafenib), which were demonstrated as safe and effective treatments for vein thrombosis $(20,21)$. Consistent with that observed in our previous studies (22-24), vein invasion, tumor number and BCLC stage were associated with OS. Notably, both univariate and multivariate analyses demonstrated that combined raltitrexed and liposomal doxorubicin could improve the OS of unresectable HCC patients. A pharmacokinetic study found that raltitrexed increased tumor cell death in a concentration- and time-dependent manner. Even with the low dose and the long infusion time, relatively high concentrations could be maintained (10). A recent retrospective study demonstrated that raltitrexedbased TACE had a longer progression-free survival time than traditional 5-fluorouracil-based TACE (25). Moreover, doxorubicin, as a liposome preparation, plays an important role in changing the pharmacokinetic properties 
and tissue distribution properties by encapsulating drugs in liposomes (26). This drug can completely penetrate the blood vessels, which creates favorable conditions for a large amount of extravasation of this drug in the lesion area. Thus, the chemotherapeutic agent can maintain a high concentration in the tumor tissue to achieve a more ideal antitumor effect (27).

The most common complication after TACE is postembolization syndrome (28). In the present study, some complications, including vomiting, myelosuppression and cardiotoxicity, were alleviated in the R + PGLD group. These effects should be attributed mainly to the PEGylated liposomes. In particular, doxorubicin enters cells through free diffusion, but it also affects the proliferation of normal dendritic cells while inhibiting the growth of tumor cells. The PEGylation of the surface of the liposome contributes to its more sustained release, in turn making it more difficult to enter the dendritic cells in the terminal growth phase of the cell. Thus, PEGylated liposomes have a more pronounced effect on reducing myelosuppression and myocardial damage (29).

There are limitations in this study. First, the sample size was not large enough. Second, this study came from a single institution. Further confirmatory studies are needed before recommending TACE with $\mathrm{R}+\mathrm{PGLD}$ as a routine treatment. Third, a few patients with BCLC A ( $\mathrm{n}=6$ and 15 in the R + PGLD and T + P groups, respectively) were included in this study because these patients with surgical contraindications could not or refused to receive surgery or radiofrequency ablation. The OS time may be affected by these patients. Fourth, so far, we did not find whether two kinds of drug combinations were more suitable for any special HCC patients, respectively. Right now, we are working for this subject. It should be an interesting and complex work because of HCC heterogeneity.

\section{Conclusions}

In summary, TACE with raltitrexed and liposomal doxorubicin could reduce the incidence of adverse reactions and significantly improve the OS of patients with unresectable HCC. In the future, additional large, randomized controlled studies are needed to evaluate this promising therapeutic approach to provide a viable option for such patients.

\section{Acknowledgments}

Funding: This research was supported by Science and
Technology Research Program of Chongqing Municipal Education Commission (No. KJQN201800416); Basic and Advanced Research Project of Science and Technology Commission of Chongqing Municipality (No. cstc2018jcyjAX0162).

\section{Footnote}

Reporting Checklist: The authors have completed the STROBE reporting checklist. Available at http://dx.doi. org/10.21037/jgo-20-59

Data Sharing Statement: Available at http://dx.doi. org/10.21037/jgo-20-59

Conflicts of Interest: All authors have completed the ICMJE uniform disclosure form (available at http://dx.doi. org/10.21037/jgo-20-59). The authors have no conflicts of interest to declare.

Ethical Statement: The authors are accountable for all aspects of the work in ensuring that questions related to the accuracy or integrity of any part of the work are appropriately investigated and resolved. The study was conducted in accordance with the Declaration of Helsinki (as revised in 2013). The study was reviewed and approved by the Ethics Review Board of the First Affiliated Hospital of Chongqing Medical University (No. 2018107). The treatment options of TACE were fully discussed with the patients and a multidisciplinary discussion group (including radiologists, surgeons, and oncologists). Written consent was obtained from each patient before treatment.

Open Access Statement: This is an Open Access article distributed in accordance with the Creative Commons Attribution-NonCommercial-NoDerivs 4.0 International License (CC BY-NC-ND 4.0), which permits the noncommercial replication and distribution of the article with the strict proviso that no changes or edits are made and the original work is properly cited (including links to both the formal publication through the relevant DOI and the license). See: https://creativecommons.org/licenses/by-nc-nd/4.0/.

\section{References}

1. Villanueva A. Hepatocellular Carcinoma. N Engl J Med 2019;380:1450-62.

2. Sia D, Villanueva A, Friedman SL, et al. Liver Cancer 
Cell of Origin, Molecular Class, and Effects on Patient Prognosis. Gastroenterology 2017;152:745-61.

3. Ricke J, Klumpen HJ, Amthauer H, et al. Impact of combined selective internal radiation therapy and sorafenib on survival in advanced hepatocellular carcinoma. J Hepatol 2019;71:1164-74.

4. Takayasu K, Arii S, Ikai I, et al. Prospective cohort study of transarterial chemoembolization for unresectable hepatocellular carcinoma in 8510 patients. Gastroenterology 2006;131:461-9.

5. Hyun MH, Lee YS, Kim JH, et al. Hepatic resection compared to chemoembolization in intermediate- to advanced-stage hepatocellular carcinoma: A meta-analysis of high-quality studies. Hepatology 2018;68:977-93.

6. Raoul JL, Forner A, Bolondi L, et al. Updated use of TACE for hepatocellular carcinoma treatment: How and when to use it based on clinical evidence. Cancer Treat Rev 2019;72:28-36.

7. Galle PR, Tovoli F, Foerster F, et al. The treatment of intermediate stage tumours beyond TACE: From surgery to systemic therapy. J Hepatol 2017;67:173-83.

8. Crocetti L, Bargellini I, Cioni R. Loco-regional treatment of HCC: current status. Clin Radiol 2017;72:626-35.

9. El-Mesallamy HO, El Magdoub HM, Chapman JM, et al. Biomolecular study of human thymidylate synthase conformer-selective inhibitors: New chemotherapeutic approach. PLoS One 2018;13:e0193810.

10. Zhu LZ, Xu S, Qian HL. Transarterial embolization and low-dose continuous hepatic arterial infusion chemotherapy with oxaliplatin and raltitrexed for hepatocellular carcinoma with major portal vein tumor thrombus. World J Gastroenterol 2018;24:2501-7.

11. Abou-Alfa GK, Johnson P, Knox JJ, et al. Doxorubicin plus sorafenib vs. doxorubicin alone in patients with advanced hepatocellular carcinoma: a randomized trial. JAMA 2010;304:2154-60.

12. Wei M, Guo X, Tu L, et al. Lactoferrin-modified PEGylated liposomes loaded with doxorubicin for targeting delivery to hepatocellular carcinoma. Int J Nanomedicine 2015;10:5123-37.

13. Tak WY, Lin SM, Wang Y, et al. Phase III HEAT Study Adding Lyso-Thermosensitive Liposomal Doxorubicin to Radiofrequency Ablation in Patients with Unresectable Hepatocellular Carcinoma Lesions. Clin Cancer Res 2018;24:73-83.

14. Kao CH, Wang JY, Chuang KH, et al. One-step mixing with humanized anti-mPEG bispecific antibody enhances tumor accumulation and therapeutic efficacy of
mPEGylated nanoparticles. Biomaterials 2014;35:9930-40.

15. Lencioni R, Llovet JM. Modified RECIST (mRECIST) assessment for hepatocellular carcinoma. Semin Liver Dis 2010;30:52-60.

16. Shen Z, Ye H, Kroger M, et al. Aggregation of polyethylene glycol polymers suppresses receptormediated endocytosis of PEGylated liposomes. Nanoscale 2018;10:4545-60.

17. Silva JP, Berger NG, Tsai S, et al. Transarterial chemoembolization in hepatocellular carcinoma with portal vein tumor thrombosis: a systematic review and meta-analysis. HPB (Oxford) 2017;19:659-66.

18. Chung GE, Lee JH, Kim HY, et al. Transarterial chemoembolization can be safely performed in patients with hepatocellular carcinoma invading the main portal vein and may improve the overall survival. Radiology 2011;258:627-34.

19. Llovet JM, Bruix J. Systematic review of randomized trials for unresectable hepatocellular carcinoma: Chemoembolization improves survival. Hepatology 2003;37:429-42.

20. Zhao Y, Wang WJ, Guan S, et al. Sorafenib combined with transarterial chemoembolization for the treatment of advanced hepatocellular carcinoma: a largescale multicenter study of 222 patients. Ann Oncol 2013;24:1786-92.

21. Mazzaferro V, Sposito C, Bhoori S, et al. Yttrium-90 Radioembolization for Intermediate-Advanced Hepatocellular Carcinoma: A Phase 2 Study. Hepatology 2013;57:1826-37.

22. Liao R, Peng C, Li M, et al. Comparison and validation of the prognostic value of preoperative systemic immune cells in hepatocellular carcinoma after curative hepatectomy. Cancer Med 2018;7:1170-82.

23. Liao R, Du CY, Gong JP, et al. HBV-DNA Load-Related Peritumoral Inflammation and ALBI Scores Predict HBV Associated Hepatocellular Carcinoma Prognosis after Curative Resection. J Oncol 2018;2018:9289421.

24. Liao R, Li DW, Du CY, et al. Combined Preoperative ALBI and FIB-4 Is Associated with Recurrence of Hepatocellular Carcinoma After Curative Hepatectomy. J Gastrointest Surg 2018;22:1679-87.

25. Cui W, Fan W, Zhang Q, et al. Comparison of two transarterial chemoembolization regimens in patients with unresectable hepatocellular carcinoma: raltitrexed plus oxaliplatin versus 5 -fluorouracil plus oxaliplatin. Oncotarget 2017;8:79165-74.

26. Rau KM, Lin YC, Chen YY, et al. Pegylated 
liposomal doxorubicin (Lipo-Dox $(\mathrm{R})$ ) combined with cyclophosphamide and 5-fluorouracil is effective and safe as salvage chemotherapy in taxane-treated metastatic breast cancer: an open-label, multi-center, non-comparative phase II study. BMC Cancer 2015;15:423.

27. Rivankar S. An overview of doxorubicin formulations in cancer therapy. J Cancer Res Ther 2014;10:853-8.

28. Dhand S, Gupta R. Hepatic transcatheter arterial

Cite this article as: Liao R, Zhang XD, Li GZ, Qin KL, Yan $\mathrm{X}$. Comparison of transcatheter arterial chemoembolization with raltitrexed plus liposomal doxorubicin $v s$. tegafur plus pirarubicin for unresectable hepatocellular carcinoma. J Gastrointest Oncol 2020;11(4):747-759. doi: 10.21037/jgo-20-59 chemoembolization complicated by postembolization syndrome. Semin Intervent Radiol 2011;28:207-11.

29. Hunault-Berger M, Leguay T, Thomas X, et al. A randomized study of pegylated liposomal doxorubicin versus continuous-infusion doxorubicin in elderly patients with acute lymphoblastic leukemia: the GRAALL-SA1 study. Haematologica 2011;96:245-52. 